\title{
EL PODER Y LOS AFECTOS EN EL ANÁLISIS DE LAS RELACIONES Y LAS TRANSFORMACIONES EN LAS FAMILIAS
}

\section{TOWARDS A COMPREHENSIVE ANALYSIS OF THE CHANGES IN FAMILIES}

\author{
Nancy Piedra Guillén ${ }^{1}$ \\ npiedraguillen@yahoo.com
}

Fecha de recepción: 21 noviembre 2014 - Fecha de aceptación: 12 agosto 2015

\begin{abstract}
Resumen
El trabajo presenta un análisis de los estudios que se han realizado en América Latina y varios países desarrollados sobre las familias. Por la prolífica producción en la temática se le da principal importancia a los textos que en las últimas tres décadas analizan las modificaciones en las familias considerando la incorporación de las mujeres al mercado laboral y enfatizan en las relaciones de poder al interior de las familias y los afectos en estas, los dos últimos temas de reciente incursión en las ciencias sociales. Este tipo de análisis observan la complejidad de la dinámica familiar que es micro social y que impacta procesos macro sociales. Estos estudios dan un giro a la perspectiva lineal que hasta el momento dominaba las investigaciones anteriores de los años 90 s en las familias, que enfatizaban en el tema de la composición de las mismas para estudiar los cambios ocurridos en las últimas décadas. Actualmente, la temática de las familias se analiza de forma más integral y multidimensional.

Palabras claves: Transformaciones familiares, Composición de las familias, Relaciones de poder en las familias, Uso del tiempo, Amor y afectos en las familias
\end{abstract}

\begin{abstract}
Abstrac
This document presents an analysis of studies of the family from Latin America, including Costa Rica, as well as some developed countries. Emphasis was given to documents from the last three decades that analyze modifications in the family, considering the incorporation of women in the changing occupational market. Emphasis was given to studies with a holistic perspective on two aspects of recent interest: power relations and affection within families. This document complements these studies and notes the complexity of this dynamic, which is micro-social but impacts macro-social processes. These contemporary studies move away from the linear perspective that has until now dominated research in families, which emphasized the issue of family composition, towards studying how families have changed in recent decades. In this way families are analyzed from a more comprehensive and multidimensional viewpoint.

Keywords: Transformations in families, Family composition, Power relations in families, use of time, Love and affection within families.
\end{abstract}

1 Profesora investigadora de la Escuela de Sociología y el Posgrado Centroamericano en Sociología, Universidad de Costa Rica, Sede Rodrigo Facio. 


\section{Introducción}

En el presente artículo se realiza una caracterización de las familias en Costa Rica a la luz de los estudios que se han realizado en América Latina y otras partes del mundo. Se van señalando particularidades de los estudios realizados en otros países y se informa sobre los estudios que se han elaborado en el país sobre el mismo. Se relaciona el tema de la familia con dos aspectos que son vitales para comprender los fenómenos de transformación macro y microsocial; que han sido poco tratados en Costa Rica esto es el tema de las relaciones de poder y los afectos en las relaciones familiares.

Con el fin de evidenciar la importancia de estudiar a las familias desde una perspectiva más integral, diversa y compleja se realiza un balance crítico de los estudios realizados en otros países, para contrastar con lo estudiado en Costa Rica y romper con una perspectiva lineal que ha predominado en los estudios de las familias yendo más allá del estudio de la conformación y la estructura de la misma. De esta forma se analiza en específico las familias y sus transformaciones, el uso del tiempo y las diferencias de género como aspectos que contribuyen a comprender los cambios y nuevos retos en las familias. Finalmente se trató el tema de las relaciones de poder y el afecto en las relaciones de pareja ${ }^{2}$.

\section{Cambios en las familias}

En las dos últimas décadas del siglo XX y la primera del XXI, se han realizado cantidad de estudios en diferentes países que dan cuenta de las transformaciones que se han generado en las familias, enfatizando en los cambios impulsados por factores externos e internos, macro y micro sociales. Aspectos como: la creciente participación de las mujeres en el mercado laboral, el aumento de su nivel educativo y su creciente participación en el mundo político. Esto favorece el cuestionamiento del modelo patriarcal de la familia nuclear tradicional, así como la organización y composición de las familias tal y como lo indican los estudios de Chafetz, 1991; Oliveira y Ariza, 1998; Wainerman, 2002; Cea, 2007, entre otros.

Oliveira y Ariza (2008, 2003, 2001 y 1997), García (2003) y García y Oliveira (2006 y 2004) conocidas por su prolífera producción en los estudios de las familias mexicanas y el impacto de los cambios socioeconómicos globales en el trabajo doméstico y extradoméstico, además de sus investigaciones sobre la vinculación de ambos en las familias y los múltiples análisis sobre aspectos varios de las familias en América Latina. Sus diversos textos enfatizan una y otra vez que aunque hay una mayor participación de las mujeres en el mercado de trabajo dicha dinámica no ha estado acompañada de una mayor participación de los varones en la reproducción doméstica, generando serios problemas a las familias en distintos ámbitos de la vida cotidiana de las mismas, incluso acentuándose prácticas violentas de parte de los hombres con respecto a sus parejas.

No es casual, por lo antes citado, que los estudios sobre las familias se nutran considerablemente de la perspectiva de género, enfoque que cuestiona las limitaciones de los estudios funcionalistas así como la visión dualista de la cultura, la naturaleza, la familia y la sociedad y enfatizan en la necesidad de considerar el mundo privado y público como una unidad, aspecto nada sencillo de superar teórica y metodológicamente (García y Oliveira, 2006 y 1994).

Varios estudios, sistemáticamente, señalan las transformaciones significativas en la estructura familiar, tales como la reducción del tamaño de las familias, el incremento en las relaciones premaritales, el aumento en el número de hogares en los que ambos cónyuges trabajan, el incremento de los hogares uniparentales (familias pluripersonal y unipersonal) (CEPAL, 1994 y 2000; Arriagada, 2007; Chant y Craske,

2 Esta reflexión forma parte de la tesis doctoral $\square$ Amor / desamor: relaciones de poder en las parejas y vínculos amorosos en la Costa Rica urbana contemporánea $\square$, en artículos posteriores se expondrá los resultados de la investigación realizada. 
2003; Robichaux, $2007^{3}$ ). Se señala que también han surgido "nuevos tipos de hogares" como la pareja de personas mayores de edad y los hogares "no nucleares". Se han constatado reducciones en las restricciones sexuales, el inicio de la vida sexual más temprano, y relacionado con el ámbito de la sexualidad se plantea el acceso a métodos anticonceptivos diversos y más accesibles (CEPAL, 1994; Arriagada, 2007; Jelin, 1998).

Las transformaciones identificadas en los procesos de formación y disolución de la familia, han dado origen a un debate en donde hay quienes hablan de una crisis del modelo de las familias nucleares biparentales y otros observan la emergencia de una familia menos patriarcal, en donde el poder es menos simétrico, aunque no desaparecen las diferencias totalmente en el interior de las mismas (Castells y Subirats, 2007). Si se parte de que la familia nuclear tal y como la se entiende hoy, se desarrolló junto con el modelo de producción capitalista, hay que considerar el impacto que tuvo dicho modelo en la división sexual del trabajo al interior de la unidad doméstica.

El "pater familia" se consolida como figura de autoridad en un grupo reducido, ejerce control y poder e incluso se sobrepone, como patriarca, a las diferencias de clase. Así, los pactos patriarcales han cumplido un papel estratégico que explica, en parte, las desigualdades de género (Izquierdo, 1998; Amorós y Cobo, 2005; Cobo, 2005). Por eso, visualizar cambios en las relaciones de poder en las familias y que se generen transformaciones en el interior de las mismas es un aspecto que debe ser estudiado, para comprender los retos y dificultades que se presentan, de tal forma que se generen procesos socioculturales trasformadores, en busca de relaciones más equitativas y democráticas en dicho espacio social.

Por ahora, los estudios señalan que en la actualidad se está dando un proceso de cambio en la estructura familiar originado por otros procesos sociales que la impactan, como la incorporación de las mujeres al trabajo extradoméstico, temática ampliamente estudiada en América Latina y otros países (Díaz et all, 2004; Flaquer, 1998; Ariza y Oliveira 2008, 2003, 2001; Wainerman, 2007; Cea, 2007; entre otros).

Ahora bien, las modificaciones no son procesos homogéneos. La complejidad abre las puertas a la diversidad; razón por la cual se habla de la existencia de distintos tipos de familias, considerando las diferencias entre los continentes y el desarrollo de las sociedades, la diversidad y el cambio observable en las familias en Europa, Canadá y Estados Unidos, a veces difiere del proceso que se verifica en los países latinoamericanos. De modo tal que, aunque la familia nuclear que es la tradicional no ha desaparecido, hay consenso en que su importancia cuantitativa disminuye en la medida en que surgen nuevas formas de vida en familia, o bien nuevos arreglos familiares como las uniones de visita, arreglos no residenciales, la cohabitación y los arreglos familiares (Beck y Beck-Gernsheim, 2001) y más recientemente las uniones formales de parejas gays y lésbicas como resultado de reivindicaciones de los colectivos en pro de la diversidad LGBTI y una mayor apertura cultural (Barrientos, Fonseca, Mora y Valverde, 2013 y Piedra, 2013).

Por otro lado, existen estudios sobre nuevas formas de organización familiar. En estos casos se examinan las diferencias en cuanto a la constitución de las familias y con respecto a las dinámicas internas que se generan en ellas. Si bien este último aspecto es el menos estudiado, hay investigaciones que han abordado el tema de los nuevos arreglos familiares, distintas formas de convivencia y el impacto de las migraciones con respecto a las familias (Jelin, 1998; Burin y Meler, 1998; Ariza y Oliveira, 2001, 2008; Cea, 2007).

Existe así, un esfuerzo sistemático y significativo en el estudio de las familias, que hablan de la necesidad de contar con un nuevo enfoque para el análisis de la estructura, composición y dinámica de los hogares o bien de las familias. A finales de la década del noventa del siglo pasado,

3 El libro de Robichaux (2007) reúne varios textos sobre el tema de las familias con el objetivo de captar y mostrar la diversidad que existe en la realidad latinoamericana. Considera que la categoría "cultura" es necesaria para captar las distintas lógicas o dinámicas que subyacen en la formación de los grupos familiares, y de ahí explicar sus morfologías. Los estudios refieren a distintos sectores socioculturales o socio clasistas de Argentina, Brasil, Cuba, Guatemala, México y Nicaragua. 
los estudios enfatizaban cada vez más la necesidad de encaminar el tratamiento de los hogares no como unidades homogéneas o como un conglomerado de personas estructuradas en torno a la figura de un jefe del hogar, sino de la existencia de diferencias tanto de edad como sexo que impactan la dinámica de las mismas.

Asimismo, se señala que las personas que integran el hogar establecen vínculos de competencia en relación con el acceso y distribución de los recursos y oportunidades que disponen para sobrellevar su vida, pero también se genera cooperación entre las personas para el logro de metas compartidas. Así que, el intento de estas autoras, y de muchas otras (Ariza y Oliveira,2008, 2003 y 2001; Seagalen, 1997; Hareven, 1990; Jelín, 1994, 1998; Dwyer y Bruce, 1988; Yanagisako, 1979 y otros), ha consistido desde hace años en construir enfoques que permitan tanto la estructura y composición, así como la dinámica de los hogares.

En Costa Rica, aunque los estudios realizados no son tan numerosos como en otros países de la región, se ha dado en distintos períodos una producción valiosa acerca del papel de las familias, de la crisis de la misma y de sus transformaciones. Los estudios de Reuben (2000 y 2001) y Vega (1999 y 2000) fueron pioneras sobre la temática de familia en Costa Rica con la utilización de datos estadísticos y censales. Particularmente, los estudios de Reuben analizan la estructura familiar de los hogares costarricenses, a partir de los datos de los censos de población de 1973, de 1983 y de la Encuesta de Hogares de 1993, lo que permite observar la estructura longitudinalmente. Siendo que los cambios más notorios se reportan después de la década del 90 y subsiguientes cuando se observa una reducción del tamaño en las familias.

Para el 2005, el tamaño promedio de las familias llegó a ser de 3.7 integrantes y la tasa de fecundidad pasó en promedio de 2,12 hijos/as en el 2003 a 1,76 hijos/as en el 2013 (INEC, 2014). En cuanto a la composición de las familias, los estudios plantean que, aunque los hogares nucleares siguen siendo mayoritarios, hay una modificación en el tanto su peso relativo cada vez es menor (Vega, 2001, 1999; Cordero; 1998; Programa Estado de la Nación, 2004; UNICEF, 20044 y Reuben, 2009, 2002, 2000 y 1992).

Merece destacarse que sigue existiendo el predominio de los hogares nucleares, lo que hace, que los estudios que tratan los cambios en las familias en Costa Rica, presten poca atención a las modificaciones, pues la estructura se mantiene estable a pesar del aumento de los divorcios que se denotan en los últimos diez años (Reuben, 2009, 2002, 2000 y 1992; Vega, 2011, 2005, 2003a y 2003b). Las familias con jefatura en condición de divorcio se duplicaron pasando de 2,0\% en 1984 a 4,2\% en el 2000 (Programa Estado de la Nación, 2004). Siendo que el divorcio forma parte de la lógica del matrimonio, ya que no es visto como un sacramento eclesiástico indisoluble (Salles y Tuirán, 1996; Marina, sf.) las uniones y separaciones legales o no, forman parte de la dinámica del cambio. Al respecto en Costa Rica se estima que la duración media del matrimonio terminado en divorcio en 1995 fue de 14,5 años la cual ha tendido a disminuir de manera muy atenuada siendo para el 2001 de 13,65 y en el 2005 de 13,24 años. Por lo tanto, se debe considerar que, las parejas que recurren a legalizar su unión y posteriormente se separan, se mantienen juntas por un período relativamente largo (Reuben, 2009).

Entonces, posiblemente se ha estimado que al no existir transformaciones significativas en la estructura organizativa de los hogares costarricenses, no existen tampoco cambios importantes en las relaciones al interior de los mismos. Sin embargo, si se deja de lado las cifras se puede observar que en las familias costarricenses se están produciendo cambios paulatinos que tiene que ver con dinámicas íntimas en donde las mujeres se destacan por tener una participación cada vez mayor y más importante en la toma de decisiones, aunque el cambio se presenta con mayor ímpetu en los hogares en donde ambos integrantes de la pareja tienen nivel educativo alto, las mujeres realizan trabajos extra domésticos y están conscientes de su condición de mujeres, de sus desventajas y sus derechos (Piedra, 2011).

4 Sandra Araya realiza un informe en el que se identifican los procesos de cambio, se caracteriza la estructura familiar y se relacionan con aspectos como la jefatura de hogar, el nivel de instrucción, la pobreza y la violencia intrafamiliar. 
A su vez, aunque la composición de la familia es mayoritariamente nuclear, resulta que, hay un aumento considerable en las separaciones y los divorcios, así que, muchas de las familias que se reportan como nucleares corresponden a una segunda o tercera unión, aspecto que los datos estadísticos no muestran. Esta es una modificación importante y cada vez más cotidiana del entorno que remite a pensar en problemas sociales que se generan de dichos procesos. Se coincide con Vega cuando señala que se necesita contar con personas más preparadas en las familias que logren sobrellevar los embates del divorcio y su experiencia por lo general muy dolorosa y cargada de estereotipos con respecto a las responsabilidades y espacios afectivos entre los hijos y las hijas y sus padre y madres, ahora divorciados o separados (Vega y Smith, 2012), se explicita la necesidad de promover procesos sanos y relaciones adecuadas entre ex parejas y de estos con sus hijos o hijas. De igual forma, se requiere propiciar procesos de acompañamiento para que en el nuevo hogar exista un ambiente de contención, respeto, afectos positivos que contribuyan al adecuado desarrollo de cada integrante. Experiencias gratas de segundas y terceras uniones existen, tal cual se puede constatar en los resultados de investigación de Piedra (2011), tal y como se verificó en los testimonios de algunas de las parejas entrevistadas.

Otro elemento relevante con respecto a las familias es el incremento de los hogares con jefaturas de mujeres, en especial los hogares con hijos e hijas y sin compañero. El estudio de la jefatura femenina ha sido relacionado y estudiado mayormente desde el fenómeno de la pobreza, distanciándose a veces de las investigaciones que se realizaban en el campo de la familia. Posteriores estudios abordaron aspectos relacionados con la dinámica al interior de las familias "jefeadas" por mujeres sin cónyuge, en donde se reportaron formas de convivencia familiar menos violentas que en aquellas donde la jefa de la familia convive con su cónyuge. Al parecer los varones sienten que han fracasado en su papel de proveedor y hacen uso de la violencia física y psicológica para reafirmar su autoridad que consideran pérdida o desplazada (Casique, 2007).

En Costa Rica recientemente se cuestiona la relación que se ha establecido entre jefatura femenina y pobreza, porque dicha percepción alimenta estereotipos de género y "victimiza" a las mujeres (Mora, 2004), por ello, se debe distinguir a las jefas económicas de las no económicas y considerar las diferencias de acuerdo a condiciones laborales de las mismas. De los datos que se presentan se desprende que son las jefas no económicas las que se encuentran en una posición más vulnerable. Este grupo se caracteriza por tener un nivel educativo menor, realizar actividades de tipo informal en el sector de servicios y de la ausencia casi total de intervención en espacios de organización social.

Por otra parte, indican los estudios que en Costa Rica, la mayoría de los hogares con hijos/as tienen a un hombre como jefe de hogar, en el 2002 eran un 64,1\% del total; en los hogares donde son las mujeres las jefas, sobresalen los tipos monoparentales nucleares y extensos (Programa Estado de la Nación, 2014; Piedra, 2007). Así que, la estructura de los hogares costarricenses con hijos/as ha cambiado, por ejemplo, los hogares biparentales con hombre proveedor y cónyuge ama de casa (BPHP) constituían el 63\% en 1990 y disminuyeron al $38 \%$ en el 2008 , mientras que los biparentales con doble proveedor (BPDP) aumentaron del 19\% al 35\% y los monoparentales con jefatura femenina (MPJF) pasaron del $18 \%$ al $27 \%$ en ese periodo. Los hogares MP con jefatura femenina tienen la mayor incidencia de pobreza $(24,9 \%)$ y los BPDP la menor (9,7\%); en los BP con un hombre proveedor se alcanza un 22,7\% (Román y Morales: 2010).

\section{El uso del tiempo y las diferencias de género}

Considerando las desigualdades que se muestran en el ámbito del trabajo productivo remunerado, es necesario estudiar las diferencias entre el trabajo reproductivo o el trabajo productivo no pagado. El análisis del uso del tiempo permite observar las disimilitudes de género tanto en el ámbito del trabajo doméstico como el extradoméstico, razón por la cual analizar esta dimensión en los estudios sobre las familias en la actualidad se vuelve un factor de suma importancia.

Así, las encuestas sobre el uso del tiempo permiten identificar el tiempo que hombres y mujeres dedican a gran cantidad de actividades que ejecutan. La gran dificultad para comprender el uso del 
tiempo radica en que las personas acostumbran realizar más de una actividad al mismo tiempo, sobre todo las que se realizan desde el ámbito reproductivo. La mayoría de actividades se realizan de forma simultánea y compartida, de ahí la dificultad de medirlas con exactitud y en todas sus dimensiones, pues por lo general se pondera una más que otras, y ello está relacionado con el valor social que se le asignan a las actividades en general: "todo lo que acontece, acontece simultáneamente" (Luhmann, 1985: 156).

Aunque las encuestas del uso del tiempo se realizan desde hace varias décadas, el análisis de las acciones es un aspecto al que se le ha prestado más atención recientemente. Los estudios más recientes contribuyen a identificar de mejor forma las diferencias y, sobre todo, las desigualdades entre hombres y mujeres con respecto al uso del tiempo en relación con el trabajo doméstico y extradoméstico (Faur, 2014). La distinción entre actividades principales y secundarias presupone un consenso social en lo que se considera como principal y secundario (no exento de carga de género), de ahí la dificultad para asir y medir todas las actividades. Las encuestas tratan de observar toda actividad, entre ellas el tiempo dedicado al cuidado personal (es un tiempo necesario), al trabajo remunerado, al estudio, a las labores domésticas y al descanso y la recreación ${ }^{5}$.

En Costa Rica, una vez realizada la Primera Encuesta del Uso del Tiempo se constata que los cambios acaecidos en espacios públicos, la incorporación de las mujeres al mercado laboral de forma significativa no ha traído consigo un cambio en la distribución de las responsabilidades domésticas, en especial, el cuido de niños/as y personas ancianas y enfermas o dependientes por condiciones de salud recaen, especialmente, en las mujeres, traduciéndose en jornadas femeninas dobles y triples (INEC 2008, Román y Morales, 2010 y Piedra, 2011). De ahí que cada vez más se ha enfatizado en la importancia que tiene estudiar el "uso del tiempo" para dar cuenta de las dificultades que ocasiona a las mujeres, así como la invisibilidad económica que se da del aporte que con su trabajo ligada a la esfera reproductiva realizan cotidianamente.

Finalmente, muy relacionado con los estudios relativos al uso del tiempo, surge el de la conciliación entre el trabajo familiar y el laboral. Los estudios, incluso las políticas sociales que se han gestado en Europa para atender el problema del recargo de las responsabilidades de las mujeres producto de su incorporación cada vez mayor al mercado de trabajo han generado un debate en los últimos años. Quienes apoyan las políticas de conciliación del tiempo de las mujeres, consideran que es una salida positiva que atiende las necesidades de las mujeres; quienes critican las políticas de conciliación que se han desarrollado indican que éstas esconden la división sexual del trabajo, y de nuevo, recae la responsabilidad del cuidado y crianza de las hijas y los hijos y de la familia en las mujeres, en lugar de generar prácticas que responsabilicen e incorporen cada vez más a los hombres en dicha actividad (Torns, 2007 y 2005; Martínez, 2007, Arriagada 2007).

Así, lo que se consideró positivo para que las mujeres se integraran al trabajo extra doméstico, por ejemplo, generar horarios flexibles más ajustados a sus necesidades; en la práctica estimula el alivianamiento de las responsabilidades en la crianza de las hijas y los hijos en los hombres. He aquí la contradicción en un tema, que trata de forma directa el problema del tiempo y el trabajo, tema que unos lo consideran nuevo y otros lo consideran un viejo tema con una nueva mirada; el viejo tema de la relación entre trabajo productivo y reproductivo (Torns, 2005).

Desde la sociología del trabajo se generaron varios estudios que, retomando parte de las preocupaciones actuales en dicha área de estudios se planteó el tema del trabajo y el tiempo considerando los avances y nuevas problemáticas desarrolladas desde los estudios de género. Por ejemplo, Torns piensa que si la lectura sobre el trabajo se hace en clave masculina por lo general significa empleo y

5 Becker, demógrafo-economista neoclásico, en 1957 abre el camino al análisis microeconómico en sus trabajos demográficos, aborda lo que ahora se conoce como economía de la familia y reconoce al trabajo doméstico como generador de productos y a la familia como una unidad de producción y consumo. "Posteriormente, en 1965, incorpora la preocupación por la discriminación y el uso del tiempo, desarrollando el planteamiento de la "Nueva Economía Doméstica" (New Home Economics)" (Pedrero, 2004: 77). 
jornada laboral; si por el contrario se lee en clave femenina, se convierte en algo más que empleo. De esta forma Torns (2007 y 2005) en sus distintos trabajos trata el tema del trabajo y el tiempo desde un enfoque de género y señala que por el tratamiento que se ha hecho de la conciliación en las políticas públicas en España este enfoque no parece ser la mejor solución para las mujeres, al menos tal cual está siendo planteada.

\section{El poder y el afecto en las relaciones de pareja}

El tema de las relaciones de pareja, el amor y el poder se ha tratado de forma esporádica por diversas corrientes analíticas. Los principales fundamentos se encuentran en la poesía y la filosofía, en menor medida en estudios historiográficos, sociológicos o psicológicos. Autores/as como Luhmann (1985) y Boltanski (2000) entre otros, presentan estudios socio históricos que permiten comprender el tema del matrimonio y la pareja humana en el tiempo, esto con el fin de comprender las modificaciones de las que han sido objeto a lo largo del mismo, enfatizando aspectos como la normatividad e institucionalidad la relación de estos con los procesos socioeconómicos del período en cuestión.

Otros estudios muestran cómo en los países desarrollados, el tema de las relaciones de poder en las parejas no es temática novedosa aunque tampoco abundante. Investigadores/as que estudian la estructura familiar, los ciclos de vida en las familias y las relaciones de pareja, han abordado el tema de las relaciones de poder en las parejas y la desigual distribución del trabajo doméstico. Trabajos como los de Blood y Wolfe (1960) fueron pioneros en esta dirección, al analizar de forma específica el vínculo entre el dinero y las relaciones de pareja, haciendo uso de la teoría de los recursos para dicho fin. Plantearon que el poder al interior de la familia se relacionaba con el nivel de ingresos y el prestigio derivado de realizar un trabajo remunerado. Por su parte, Alberdi (2001) y Flaquer (1998) aportan nuevos hallazgos analizando los cambios en la estructura de las familias que tiene como causa directa el aumento significativo de las parejas de doble ingreso.

Sin embargo, recientes investigaciones en Estados Unidos, Canadá y Europa (Alemania, Suecia, España y Francia, entre otros) señalan que las desigualdades persisten al interior de las familias y que, en alguna medida, encubren de forma consciente o inconsciente las relaciones de género tradicionales. En otras palabras, a pesar del creciente acceso de las mujeres a los recursos económicos, materiales, educativos y políticos, se señala que en las familias se continúa promoviendo el poder y la dominación masculina.

El estudio comparativo que se realizó entre Alemania, España, Estados Unidos y Suecia sobre las relaciones de poder en las parejas señala que, no obstante las diferencias culturales e institucionales entre esos países, el ideal de la pareja simétrica está presente pero las prácticas muestran lo distantes que están las parejas de tener relaciones igualitarias entre hombres y mujeres (Nyman y Reinikainen (2001), (Díaz et al., 2004 y Díaz et. All. 2004b), Dema (2006). En las parejas heterosexuales se constata que las mujeres no han logrado el mismo nivel de independencia y autonomía que los hombres. Tal y como lo indican las investigaciones realizadas, la idea de la igualdad de género en las relaciones de pareja queda más circunscrita al ámbito del discurso y de la intencionalidad mediada por el afecto entre las personas que conforman la pareja (Piedra, 2011).

Por su parte, estudios como los de Beck y Beck-Gernsheim (2001) y Giddens (1992) enfatizan el cambio que viven las personas en sus relaciones amorosas en la época contemporánea. En sus textos, los autores coinciden en que la transformación tiene como fundamento la práctica que se ha desarrollado en las últimas tres décadas y en la cosmovisión de las personas al considerar que la unión de una pareja - al menos en las sociedades occidentales- institucionalizada en el matrimonio, si bien sigue siendo válida, no es para toda la vida. Giddens (1992) nos refiere al tema de la transformación de la intimidad sobre las relaciones de pareja, permite entender el mundo de la psique: los deseos, las necesidades, al fin de cuentas la subjetividad y las diferencias entre hombres y mujeres para establecer una relación de pareja, relacionándolo a su vez con los cambios sociales acaecidos en occidente en las últimas décadas. 
Considera que en la actualidad enfrentamos transformaciones en la vida íntima en donde las mujeres se han destacado por su protagonismo y por la búsqueda de relaciones más democráticas, aspectos que se evidencian con mayor claridad en los países desarrollados.

Los estudios con una orientación más psicoanalítica son trabajos que consideran los aspectos específicos del yo interno relacionados con aspectos socioculturales del entorno en el que se desarrolla la relación para contextualizar las relaciones y entender sus particularidades como los trabajos realizados por Campusano (2001) y Giddens (1992).

En América Latina los estudios que tratan el tema de la negociación y los acuerdos entre la pareja son escasos. Investigaciones de esta índole se han realizado especialmente en Estados Unidos y otros países desarrollados. El referente principal para América Latina son los trabajos de Coria (2001, 1997) Díaz-Loving y Sánchez (2002), Díaz-Loving y Rivera (2002), quienes parten de un enfoque psicosocial.

Desde esta línea teórica, Díaz-Loving y Rivera (2002) y Díaz-Loving y Sanchez (2002) realizan investigaciones de carácter psicométrico en el que analizan los comportamientos de personas que cuentan con pareja. Se inclinan por medir conductas haciendo uso de regresiones o bien correlación entre variables; posteriormente, con base en la medición se establecen modelos teóricos para estudiar las prácticas y la visión que tienen las personas. Logran establecer qué factores interfieren más y tienen significancia estadística en aspectos relativos al poder y al amor. Sus estudios identifican seis tipos de amor: el pragmático, el amistoso, el maníaco, el ágape, el lúdico y el Eros.

En México y América Latina también es perceptible la ausencia de estudios que den cuenta de la importancia relativa de diferentes factores sobre el acceso a recursos, la vivencia de roles masculinos y femeninos y diversas facetas de las relaciones de poder entre hombres y mujeres. Para superar esta limitación se ha sugerido realizar estudios con enfoques multivariados a nivel estadístico que permitan especificar el peso relativo de los diferentes factores intervinientes en la explicación (Oliveira, 1998 y García y Oliveira, 2006).

Resientes resultados de investigación en Costa Rica toman en cuenta el afecto como un elemento fundamental que debemos tomar en cuenta para comprender las diferencias y las relaciones de poder que se dan en la pareja, sin duda se constata que las prácticas de poder están determinadas por los afectos y en nombre del amor y de la confianza que se debe generar del mismo. En las parejas heterosexuales quienes más ceden en la toma de decisiones en temas estratégicos para la familia o bien la pareja, como es en el uso del dinero y decisiones de envergadura, son las mujeres; porque con mayor facilidad toman una actitud más comprensiva aunque no estén de acuerdo con la decisión final. Sin embargo, tanto en parejas homosexuales como heterosexuales la persona que gana más dinero y/o tiene más recursos económicos y materiales suele incidir más en las decisiones estratégicas de la pareja o la familia (Piedra, 2011).

\section{Reflexión final}

Recapitulando, la revisión bibliográfica efectuada y considerando aspectos socio económico se observa algunas tendencias importantes:

Que las transformaciones han incidido en el modelo ideal de familia, el nuclear, restándole importancia. Dicho modelo ha cambiado en la práctica, adecuándose a los procesos sociales, económicos y culturales que se vive en las sociedades contemporáneas, muy especialmente, debido a la creciente autonomía económica de las mujeres, al aumento de los divorcios y las separaciones, a la pérdida de influencia de la iglesia en la vida civil y política y a una tendencia general hacia el individualismo en todos los ámbitos de la vida social y económica. A su vez, la legalidad o ilegalidad de la unión de la pareja pierde importancia, simbólicamente tiene más relevancia el grado de compromiso con que los integrantes asumen su relación.

Los estudios en el ámbito latinoamericano y en los países desarrollados indican que la participación de las mujeres en el mercado de trabajo, en los espacios político-organizativos y el incremento en el nivel educativo, pueden conducir a relaciones de mayor igualdad pero no son determinantes ni 
automáticos. El caso sueco -en donde las diferencias entre hombres y mujeres en el mercado de trabajo no son tan grandes como en otros países occidentales, ni en lo que se refiere al nivel de participación en el empleo ni en los salarios percibidos- es un referente importante en este sentido.

Sobre el tema de las relaciones de poder en la pareja, los estudios se han limitado, principalmente, a identificar la presencia del poder y el ejercicio del mismo por parte de los hombres, pero se ha problematizado muy poco el tema, solo se registra una investigación en esa tesitura (Piedra, 2011) en la que se analizan las representaciones simbólicas y las estrategias que los varones están adoptando, ya sea para favorecer o para bloquear posibles cambios que emanan de las iniciativas tomadas por las mujeres.

Por otra parte, en investigaciones recientes (de los $90 \mathrm{~s}$ al presente) por lo general se ha señalado que la educación y la participación laboral de las mujeres son recursos que permiten modificar en una dirección más igualitaria las relaciones de poder en las parejas. Sin embargo, en las investigaciones de la última década realizadas en Europa, Estados Unidos, Canadá y Costa Rica se plantea que la relación entre ambas variables no siempre deviene en una relación causal, la realidad es más compleja y aunque se genera cierta autonomía, no siempre se revierte en un mayor ejercicio del poder en la pareja o que el mismo sea más simétrico tanto en las familias como en las parejas (Díaz et al., 2004 y Díaz Capitolina; Díaz Cecilia, Dema e Ibáñez, 2004b y Piedra, 2011).

A su vez, los trabajos de investigación en Latinoamérica dan cuenta de las transformaciones que se están generando en la forma de organización de las familias producto de procesos de cambio estructural. Esto es relevante en tanto las investigaciones escudriñan poco en dichas transformaciones.

Estudiar el poder considerando el amor en la relación de pareja, permite realizar un análisis más completo y complejo de la relación de pareja. Estudiar la pareja y entrevistar a ambas partes que forman la misma, posiblemente, sea el principal aporte del presente trabajo para el caso costarricense e incluso latinoamericano, pues la mayoría de los trabajos revisados omiten este aspecto, entrevistan por lo general sólo a las mujeres, los estudios de paternidad entrevistan o encuestan sólo a los hombres y en caso de que se entrevisten en un mismo estudio a hombres y mujeres, no son integrantes de la misma pareja. Investigaciones recientes realizadas en Estados Unidos, Canadá y Europa apuntan en esa dirección metodológica, y se plantean el tema de la afectividad como uno de los factores que incide en el ejercicio del poder en las relaciones de pareja y en la independencia/dependencia de las mujeres.

En Costa Rica existen estudios sobre las familias, su estructura, su institucionalidad y los divorcios, pero pocos indagan sobre las parejas y las relaciones de género y, menos aún, sobre la toma de decisiones y el ejercicio del poder al interior de las unidades domésticas. Por ello, se estima que esta investigación contribuye a la discusión de cuán democráticas son las relaciones de género al interior de la parejas, al menos desde el espacio intrafamiliar, relativizando o confirmando, con ello, los notorios logros que en materia de equidad e igualdad de género revela la evolución de los índices de Naciones Unidas antes citados.

En Costa Rica los estudios, en su mayoría, giran en torno a un tópico muy específico de las familias, y en ninguno de los casos se plantea el tema de las transformaciones que se están suscitando en la sociedad contemporánea en torno a las prácticas de poder que tienen lugar entre ambos integrantes de la pareja. Por otra parte, son escasos los estudios realizados en Costa Rica para analizar las familias desde una perspectiva de género, a no ser que éstas se refieran a la participación de las mujeres en el mercado laboral o bien a temas relacionados con la violencia intrafamiliar y, más recientemente, sobre la paternidad responsable. Por lo general, los trabajos toman como base para el análisis de los cambios y la diversidad en las familias los datos estadísticos, así como aspectos normativos y legislativos. Tal es el caso de la "Ley de la paternidad responsable".

\section{Bibliografía}

Amorós, Celia y Cobo, Rosa. (2005). Feminismo e ilustración. En: Celia Amorós y Ana de Miguel (2005). Teoría feminista: de la ilustración a la globalización. De la ilustración al segundo sexo. Madrid, Minerva Ediciones. 
Alberdi, Inés (2001). Padres, madres e hijos ante los nuevos valores de la familia española. En: Durán et.al. Estructura y cambio social. Homenaje a Salustiano del Campo, Madrid, CIS.

Ariza, Marina y Oliveira, Orlandina (2008). Familias, pobreza y desigualdad social en Latinoamérica: una mirada comparativa. En: Rev. Latinoamericana de Población, Asociación Latinoamericana de Población, año 1, núm. 2, enero-junio pp. 73-98.

Ariza, Marina y Oliveira, Orlandina (2003). Acerca de las familias y los hogares: estructura y dinámica. En: Wainerman, Catalina (ed.). Familia, trabajo y género: un mundo de nuevas relaciones. Buenos Aires, Fondo de Cultura Económica, Fondo de las Naciones Unidas para la Infancia (UNICEF).

Ariza, Marina y Oliveira, Orlandina (2001). Familias en transición y marcos conceptuales en redefinición. Toluca. Papeles de Población, Universidad Autónoma del Estado de México. Toluca.

Arriagada, Irma. (2007). Familias latinoamericanas: cambiantes, diversas, desiguales. Rev. Papeles de Población, julio-septiembre, núm. 53, Universidad Autónoma del Estado de México, Toluca. pp. 9-22.

Arriagada, Irma. (2002) Cambios y desigualdad en las familias latinoamericanas. Rev. de la CEPAL, 77:143-61.

Barrientos, Adrian et all. (2013). Análisis de las transformaciones en la estructura y conformación de los hogares y familias en Costa Rica la luz de los Censos de Población y Vivienda del 2000 y 2011. Tesis de licenciatura, Universidad de Costa Rica.

Beck, U. y G. Beck-Gernsheim. (2001). El normal caos del amor, las nuevas formas de la relación amorosa, Barcelona, Paidós Contextos - El Reure.

Blood, Robert y Wolfe, Donald. (1960). Husband and wives, Nueva York, Free Press.

Boltanski, Luc. (2000). El amor y la justicia como competencia, tres ensayos de sociología de la acción, Buenos Aires, Amorrortu/editores.

Burin, Mabel y Meler, Irene. (1998). Género y familia, poder, amor y sexualidad en la construcción de la subjetividad. Buenos Aires, Barcelona y México, Paidós.

Casique, Irene. (2007). Multiplicidad del vínculo entre empoderamiento de la mujer y la violencia de género. En: III Encuentro Nacional de Demógrafos y Estudiosos de la Población, 2007. Cambios Demográficos en Venezuela: Oportunidades y Retos para las Políticas Públicas, Venezuela.

Castells, Manuel y Subirats, Marina. (2007). Mujeres y hombres ¿un amor imposible?, Madrid, Alianza Editorial.

Cea, María de los Ángeles. (2007). La deriva del cambio familiar, Madrid, Centro de Investigaciones Sociológicas.

Cea, María de los Ángeles. (2000). Mujer y trabajo en las reformas estructurales latinoamericanas durante las décadas de 1980 y 1990. Septiembre de 2000, Serie Mujer y Desarrollo Nro. 28.

Chafetz, Janet. (1991). The Gender Division of Labor and the Reproduction of Female Disadvantage: Toward an Integrated Theory. En: Rae Lesser Blumberg (ed.) Gender, Family and Economy. Sage, Newbury Park, 1991, pp. 74-94.

Cobo, Rosa. (2005). El género en las ciencias sociales. Rev. Cuadernos de Trabajo Social. Vol. 18, pp. 249- 258.

Coria, Clara. (2001). EL AMOR, no es como nos contaron... ni como lo inventamos, Buenos Aires, Paidós.

Coria, Clara. (1997). Las negociaciones nuestras de cada día, México D.F., Paidós.

Dema, Sandra. (2006). Pareja, dos salarios. El dinero y las relaciones de poder en las parejas del doble ingreso. Madrid, Centro de Investigaciones sociológicas.

Díaz, Capitolina, Díaz Méndez, Cecilia, Dema, Sandra, e Ibáñez, Marta. (2004). Dinero, amor e individualización: las relaciones económicas en las parejas/familias contemporáneas. Oviedo, Instituto Asturiano de la Mujer. KRK Ediciones.

Díaz, Rolando y Sánchez Rozzana. (2002). Psicología del amor: una visión integral de la relación de pareja. México D.F., Facultad de Psicología UNAM y Miguel Ángel Porrúa. 
Díaz-Loving y Rivera, Sofía. (2002). La cultura del poder en la pareja, México D.F., Miguel Ángel Porrúa.

Dwyer, Daisy y Judith Bruce (ed.) (1988). A Home Divided. Women and Income in the Third World. Stanford, Stanford University Press, pp. 1-19.

Faur, Eleonor (2014). El cuidado infantil en el siglo XXI: Mujeres malabaristas en una sociedad desigual. Buenos Aires, Siglo Veintiuno Editores.

Fernández, Juan Antonio y Constanza Soler. (2005). Conciliar las responsabilidades familiares y laborales; políticas y prácticas sociales. Documento de trabajo $\mathrm{N}^{\circ} 79$, fundación Alternativas.

Flaquer, Lluís. (1998). El destino de la familia, Barcelona, Ariel.

García, Brígida y Oliveira, Orlandina. (2006). Las familias en el México Metropolitano: visiones femeninas y masculinas, México D.F., El Colegio de México.

García, Cristina y Carrasco, Cristina. (2005). El tiempo, los tiempos, una vara de desigualdad. Chile, CEPAL, Serie Mujer y Desarrollo núm. 65.

Giddens, Anthony (1992). La transformación de la intimidad, sexualidad, amor y erotismo en las sociedades modernas, España, Cátedra.

Hareven, Tamara. (1990). A Complex relationship: family strategies and the process of economic social change. En: Roger Friedland y A.F. Robertson (eds.), en Beyond the Market Place, Rethinking Economy and Society, New York, Aldine de Grunter, pp. 215-244.

Instituto Nacional de Estadística y Censo INEC. (2008). Principales resultados del Módulo de Uso del Tiempo, San José C.R. Serie de Estudios Especiales.

Instituto Nacional de Estadística y Censo INEC. (2014). Datos en Línea.

Izquierdo, María Jesús. (1998). El malestar en la desigualdad, Madrid, Ediciones Cátedra. Feminismos.

Jelin, Elizabeth. (1998). Pan y afectos, transformación de las familias, México, Fondo de Cultura Económica.

Luhmann, Niklas. (1985). El amor como pasión, la codificación de la intimidad. Barcelona, Ediciones Península.

Marina, José Antonio. (s.f). La familia: un modelo para armar. Recuperado de http://www.movilizacioneducativa.net/pdf/La\%20familia.\%20un\%20modelo\%20para\%20armar.pdf.

Martínez, Juliana. (2007). La pieza que faltaba: uso del tiempo y regímenes de bienestar en América Latina”, Revista Nueva Sociedad, núm. 199, Sección Aportes.

Mora, Lorena y Ugalde K. (2008). El ejercicio de la paternidad, calidad de vida y construcción del proyecto de vida en adolescentes urbanos. Tesis de Licenciatura en Psicología, Universidad de Costa Rica.

Mora, Minor. (2004). Visión crítica del vínculo entre jefatura de hogar, estratificación y análisis de clase. En: Rev. Ciencias Sociales, Universidad de Costa Rica núm. 105, 2004 (III).

Nyman, Charlott y Reinikainen, Lasse. (2001). Bajo la aparente igualdad de género, el reparto del dinero en las familias suecas. Rev. Abaca: Cultura y Ciencias Sociales, núm. 29-30, pp. 29-38.

Oliveira, Orlandina. (2007). "Reflexiones acerca de las desigualdades sociales y el género". Rev. Estudios Sociológicos, vol. XXV, núm. 75, El Colegio de México, septiembre-diciembre, pp. 805-812.

Pedrero, Mercedes. (2004). Género, trabajo doméstico y extradoméstico en México. Una estimación del valor económico del trabajo doméstico. En: Rev. Estudios Demográficos y urbanos, vol. 19, núm. 2 (56), mayo-agosto, pp. 413-446.

Piedra, Nancy. (2011). Amor y desamor: relaciones de poder en la Costa Rica contemporánea. Tesis de doctorado. El Colegio de México. México.

Programa de las Naciones Unidas para el Desarrollo PNUD y Organización Internacional del Trabajo OIT. (2009). Trabajo y Familia: Hacia nuevas formas de conciliación con corresponsabilidad social. Santiago de Chile, Oficina Internacional del Trabajo y Programa de las Naciones Unidas para el Desarrollo. 
Programa Estado de la Nación. (2004). Décimo Informe Estado de la Nación en Desarrollo Humano Sostenible, San José, Costa Rica.

Reuben, Sergio. (2009). Duración media del matrimonio terminado en divorcio. Informe de trabajo del "Proyecto de investigación No. 211-A8-700". Escuela de Sociología de la Universidad de Costa Rica.

Reuben, Sergio. (2002). El carácter histórico de la familia y las transformaciones sociales contemporáneas. En: Rev. Reflexiones de la Facultad de Ciencias Sociales de la Universidad de Costa Rica, Vol. 81, núm. 2, 2002.

Reuben, Sergio. (2001). "Característica familiares de los hogares costarricenses”. En: Cristina Gomes (Compiladora), Procesos sociales, población y familia. México, FLACSO, p. 147-198.

Reuben, Sergio. (2001). "Política social y familia". En: Rosero, Luis. Población del Istmo 2000: Familia, migración, violencia y medio ambiente, Centro Centroamericano de Población, Universidad de Costa Rica, p. 25-40.

Reuben, Sergio. (2000). Política social y familia. En: Contrapunto, No .8 , Sinart, San José, 2000, p. 15-21.

Román, Isabel y Morales; Natalia. (2010). Demanda potencial de cuido infantil según estructura de los hogares: el caso de Costa Rica. Informe de Trabajo. Disponible en página web del Instituto Nacional de las Mujeres.

Salles, Vania y Tupirán, Rodolfo. (1996). Mitos y creencias sobre la vida familiar. En: Rev. Mexicana de Sociología. Año LIVIA, vol. 2.

Seagalen, Martine. (1997). Antropología histórica de la familia, Madrid, Taurus Ediciones, pp. 123-142.

Torns, Teresa. (2007). El trabajo, género y tiempo social. En: Prieto, Carlos (ed.) Trabajo, género y tiempo social, Madrid, Ediciones Gráficas Arial, 2007 p. 111-117.

Torns, Teresa. (2005). De la imposible conciliación a los permanentes malos entendidos. En, Madrid, Cuadernos de Relaciones laborales, 2005, 23, núm. 1, p. 15-33.

Torns, Teresa. (2004). Las políticas de tiempo: un reto para las políticas del Estado de Bienestar. Trabajo. Revista de la Asociación Estatal de Centros Universitarios de RR.LL y de CC.TT (enero-junio) p.145-164.

UNICEF. (2004). Desafíos y retos de las familias costarricenses. IV Estado de los Derechos de la Niñez y la Adolescencia en Costa Rica. UNICEF, Universidad de Costa Rica. San José, 25p.

Vega, Isabel. (2011). Papás divorciados sin la custodia de sus hijos e hijas. Ficciones y realidades. San José, Instituto de Investigaciones Psicológicas, Universidad de Costa Rica.

Vega, Isabel. (2005). Paternidad y divorcio: Una aproximación desde las vivencias de 5 padres costarricenses. En J.R. Martínez y Mira, R. (Eds.). Psicología Social y problemas sociales (pp. 549-557). Madrid: Editorial Biblioteca Nueva, S.L.

Vega, Isabel. (ed.) (2003a). Pareja y familia en la sociedad actual: ¿nuevos significados y desafíos? San José, Editorial Universidad de Costa Rica.

Vega, Isabel. (2003b). El divorcio y las nuevas dimensiones de la paternidad, San José, Editorial Universidad de Costa Rica.

Vega, Isabel. (2001). Las familias costarricenses en el contexto del nuevo milenio. En: Realidad familiar en Costa Rica, San José, FLACSO, UNICEF, Instituto de Investigaciones Psicológicas, Universidad de Costa Rica.

Vega, Isabel y Smith Vania. (2009). Correlatos y predictores del cumplimiento de pago de la pensión alimentaria en padres divorciados o separados. En: Rev. Interamericana de Psicología, 43 (2), 395-404.

Vega, Isabel y Smith Vania. (2012). Desarrollo y validación de la escala de creencias sobre el rol paterno posdivorcio en padres costarricenses divorciados. En: Acta Colombiana de Psicología, vol. 15, núm. 2, 2012, pp. 65-74.

Villarreal, Ana. (1999). Relaciones de poder, mujeres en la encrucijada entre trabajo productivo y trabajo reproductivo. Posgrado en Sociología, Universidad de Costa Rica, tesis de maestría. 
Yanagisako, Silvia Junko. (1979). Family and Household: the Analysis of Domestic Groups. Rev. Annual Review of Anthropology, núm. 8, pp. 161-204.

Wainerman, Catalina. (2007). Conyugalidad y paternidad ¿Una revolución estancada? En: Género familias y trabajo: rupturas y continuidades. Desafíos para la investigación política, Gutiérrez, María Alicia, Buenos Aires, CLACSO, Consejo Latinoamericano de Ciencias Sociales. 
